\title{
The semi-constrained NMSSM satisfying bounds from the LHC, LUX and Planck
}

\author{
Ulrich Ellwanger $^{a}$ and Cyril Hugonie ${ }^{b}$ \\ ${ }^{a}$ LPT, UMR 8627, CNRS, Université de Paris-Sud, \\ Bât. 210, 91405 Orsay, France \\ ${ }^{b}$ LUPM, UMR 5299, CNRS, Université de Montpellier II, \\ Place Eugène Bataillon, 34095 Montpellier, France \\ E-mail: ulrich.ellwanger@th.u-psud.fr, cyril.hugonie@um2.fr
}

\begin{abstract}
We study the parameter space of the semi-constrained NMSSM, compatible with constraints on the Standard Model like Higgs mass and signal rates, constraints from searches for squarks and gluinos, a dark matter relic density compatible with bounds from WMAP/Planck, and direct detection cross sections compatible with constraints from LUX. We consider the region in parameter space with an additional lighter Higgs boson in the 60-120 GeV mass range. It is detectable in the diphoton mode or in decays into a pair of lighter CP-odd Higgs bosons. This region in parameter space allows for a fine-tuning as low as about 100, and dominantly singlino like dark matter with a mass down to $1 \mathrm{GeV}$, but possibly a very small direct detection cross section.
\end{abstract}

KEYWORDS: Supersymmetry Phenomenology

ARXIV EPRINT: 1405.6647 


\section{Contents}

1 Introduction $\quad 1$

2 The NMSSM with constraints at the GUT scale 2

3 Impact of LHC constraints on squark/gluino masses and fine-tuning 5

4 Properties of dark matter $\quad 8$

5 Properties of the lighter Higgs boson $H_{1} \quad 10$

$\begin{array}{llr}6 & \text { Conclusions and outlook } & 13\end{array}$

\section{Introduction}

Recent results from the LHC and direct dark matter detection experiments constrain considerably possible scenarios beyond the Standard Model (SM), amongst others its supersymmetric (SUSY) extensions. These constraints originate essentially from the Higgs mass $[1,2]$ and its quite SM like signal rates, the absence of signals in searches for squarks and gluinos after the $8 \mathrm{TeV}$ run at the LHC [3, 4], and upper bounds on dark matter-nucleus cross sections from the LUX experiment [5].

Masses and couplings of Higgs boson(s), SUSY particles and notably the lightest SUSY particle (LSP, the dark matter candidate), are strongly correlated in SUSY extensions of the SM if one assumes at least partial unification of the soft SUSY breaking terms at a grand unification (GUT) scale. Hence it is interesting to study how the combined constraints affect the parameter space and, notably, which signals beyond the SM we can expect in the future. Such studies (after the discovery of the $126 \mathrm{GeV}$ Higgs boson) had been performed earlier in the Minimal SUSY extension of the SM (MSSM) [6-26] and the Next-to-Minimal SUSY extension of the SM (NMSSM) [7, 24, 27-31].

These studies differ, however, in the treatment of the soft SUSY breaking terms in the Higgs sector at the GUT scale: in "fully constrained" versions of the MSSM or NMSSM these are supposed to be unified with the soft SUSY breaking terms in the squark and slepton sectors. In NUHM (non-universal Higgs masses) or "semi-constrained" versions of the MSSM or NMSSM one allows the soft SUSY breaking terms in the Higgs sector to be different; after all the quantum numbers of the Higgs fields differ from those of quarks and leptons: Higgs fields are in a real representation $(2+\overline{2})$ of $\mathrm{SU}(2)$, but do not fit into complete representations of SU(5); these properties can easily have an impact on the presently unknown sources of soft SUSY breaking terms. In the NMSSM including the singlet superfield $S$, "semi-constrained" can indicate non-universal soft SUSY breaking 
terms involving the singlet only, or non-universal soft SUSY breaking terms involving SU(2) doublet or singlet Higgs fields. In the present study we allow for the latter more general case.

Previous studies of the NMSSM with constraints at the GUT scale [24, 27-31] had found that wide ranges of parameter space comply with constraints from the LHC and on dark matter, and that less "tuning" is required than in the MSSM $[24,30]$. These findings are confirmed by scans of the parameter space of the general NMSSM (without constraints at the GUT scale) [32-38], and motivate a thorough analysis of the semi-constrained NUHNMSSM with up-to-date experimental constraints, amongst others on Higgs signal rates and bounds on dark matter-nucleus cross sections [5]. "NUH" appears without "M" since, apart from the Higgs mass terms, also trilinear couplings involving Higgs bosons only are allowed to differ from trilinear couplings involving squarks or sleptons at the GUT scale, see the next section.

Using the code NMSPEC [40] within NMSSMTools_4.2.1 [41, 42] together with micrOMEGAS_3 [43] we have sampled about 3.2 M viable points in the parameter space, which allows us to cover the complete range of masses and couplings of the LSP and additional Higgs bosons, parts of which had not been observed in previous analyses. In this paper we confine ourselves to regions where an additional NMSSM-specific Higgs scalar is lighter than the SM-like Higgs boson near $126 \mathrm{GeV}$; this region is strongly favoured by the mass of the SM-like Higgs boson, and contains the most interesting phenomena to be searched for in the future.

In the next section we present the model, the applied phenomenological constraints, the definition of fine-tuning, and the ranges of parameters scanned over. In section 3 we discuss the impact of unsuccessful searches for squarks and gluinos at the LHC on fine-tuning and some of the parameters like the soft squark/slepton masses $m_{0}$, the universal gaugino masses $M_{1 / 2}$ and the NMSSM-specific Yukawa coupling $\lambda$. Section 4 is devoted to the properties of the LSP, its detection rates to be expected in the future, and its annihilation processes allowing for a viable relic density. In section 5 we discuss the Higgs sector, in particular prospects to detect the lighter NMSSM specific Higgs scalar. Conclusions and an outlook are given in section 6 .

\section{The NMSSM with constraints at the GUT scale}

The NMSSM [44] differs from the MSSM due to the presence of the gauge singlet superfield $S$. In the simplest $\mathbb{Z}_{3}$ invariant realisation of the NMSSM, the Higgs mass term $\mu H_{u} H_{d}$ in the superpotential $W_{\text {MSSM }}$ of the MSSM is replaced by the coupling $\lambda$ of $S$ to $H_{u}$ and $H_{d}$ and a self-coupling $\kappa S^{3}$. Hence, in this simplest version the superpotential $W_{\text {NMSSM }}$ is scale invariant and given by

$$
W_{\mathrm{NMSSM}}=\lambda \hat{S} \hat{H}_{u} \cdot \hat{H}_{d}+\frac{\kappa}{3} \hat{S}^{3}+\ldots,
$$

where hatted letters denote superfields, and the ellipses denote the MSSM-like Yukawa couplings of $\hat{H}_{u}$ and $\hat{H}_{d}$ to the quark and lepton superfields. Once the real scalar component of $\hat{S}$ develops a vev $s$, the first term in $W_{\text {NMSSM }}$ generates an effective $\mu$-term

$$
\mu_{\mathrm{eff}}=\lambda s .
$$


The soft Susy breaking terms consist of mass terms for the Higgs bosons $H_{u}, H_{d}, S$, squarks $\tilde{q}_{i} \equiv\left(\tilde{u}_{i L}, \tilde{d}_{i L}\right), \tilde{u}_{i}^{c}, \tilde{d}_{i R}^{c}$ and sleptons $\tilde{\ell}_{i} \equiv\left(\tilde{\nu}_{i L}, \tilde{e}_{i L}\right)$ and $\tilde{e}_{i}^{c}$ (where $i=1,2,3$ is a generation index):

$$
\begin{aligned}
-\mathcal{L}_{0}= & m_{H_{u}}^{2}\left|H_{u}\right|^{2}+m_{H_{d}}^{2}\left|H_{d}\right|^{2}+m_{S}^{2}|S|^{2}+m_{\tilde{q}_{i}}^{2}\left|\tilde{q}_{i}\right|^{2}+m_{\tilde{u}_{i}}^{2}\left|\tilde{u}_{i}^{c}\right|^{2}+m_{\tilde{d}_{i}}^{2}\left|\tilde{d}_{i_{R}}^{c}\right|^{2} \\
& +m_{\tilde{\ell}_{i}}^{2}\left|\tilde{\ell}_{i}\right|^{2}+m_{\tilde{e}_{i}}^{2}\left|\tilde{e}_{i}^{c}\right|^{2}
\end{aligned}
$$

trilinear interactions involving the third generation squarks, sleptons and the Higgs fields (neglecting the Yukawa couplings of the two first generations):

$$
\begin{aligned}
-\mathcal{L}_{3}= & \left(h_{t} A_{t} Q \cdot H_{u}{\tilde{u_{3}}}_{R}^{c}+h_{b} A_{b} H_{d} \cdot Q{\tilde{d_{3}}}_{R}^{c}+h_{\tau} A_{\tau} H_{d} \cdot L{\tilde{e_{3}}}_{R}^{c}\right. \\
& \left.+\lambda A_{\lambda} H_{u} \cdot H_{d} S+\frac{1}{3} \kappa A_{\kappa} S^{3}\right)+ \text { h.c. },
\end{aligned}
$$

and mass terms for the gauginos $\tilde{B}$ (bino), $\tilde{W}^{a}$ (winos) and $\tilde{G}^{a}$ (gluinos):

$$
-\mathcal{L}_{1 / 2}=\frac{1}{2}\left[M_{1} \tilde{B} \tilde{B}+M_{2} \sum_{a=1}^{3} \tilde{W}^{a} \tilde{W}_{a}+M_{3} \sum_{a=1}^{8} \tilde{G}^{a} \tilde{G}_{a}\right]+\text { h.c. }
$$

In constrained versions of the NMSSM one assumes that the soft Susy breaking terms involving gauginos, squarks or sleptons are identical at the GUT scale:

$$
\begin{aligned}
M_{1} & =M_{2}=M_{3} \equiv M_{1 / 2}, \\
m_{\tilde{q}_{i}}^{2} & =m_{\tilde{u}_{i}}^{2}=m_{\tilde{d}_{i}}^{2}=m_{\tilde{\ell}_{i}}^{2}=m_{\tilde{e}_{i}}^{2} \equiv m_{0}^{2}, \\
A_{t} & =A_{b}=A_{\tau} \equiv A_{0}
\end{aligned}
$$

In the NUH-NMSSM considered here one allows the Higgs sector to play a special role: the Higgs soft mass terms $m_{H_{u}}^{2}, m_{H_{d}}^{2}$ and $m_{S}^{2}$ are allowed to differ from $m_{0}^{2}$ (and determined implicitely at the weak scale by the three minimization equations of the effective potential), and the trilinear couplings $A_{\lambda}, A_{\kappa}$ can differ from $A_{0}$. Hence the complete parameter space is characterized by

$$
\lambda, \kappa, \tan \beta, \mu_{\mathrm{eff}}, A_{\lambda}, A_{\kappa}, A_{0}, M_{1 / 2}, m_{0},
$$

where the latter five parameters are taken at the GUT scale.

Expressions for the mass matrices of the physical CP-even and CP-odd Higgs states after $H_{u}, H_{d}$ and $S$ have assumed vevs $v_{u}, v_{d}$ and $s$ and including the dominant radiative corrections - can be found in [44] and will not be repeated here. The physical CP-even Higgs states will be denoted as $H_{i}, i=1,2,3$ (ordered in mass), and the physical CP-odd Higgs states as $A_{i}, i=1,2$. The neutralinos are denoted as $\chi_{i}^{0}, i=1, \ldots, 5$ and their mixing angles $N_{i, j}$ such that $N_{1,5}$ indicates the singlino component of the lightest neutralino $\chi_{1}^{0}$.

Subsequently we are interested in regions of the parameter space where doublet-singlet mixing in the Higgs sector leads to an increase of the mass of the SM-like (mostly doubletlike) Higgs boson, which leads naturally to a SM-like Higgs boson $H_{2}$ in the $126 \mathrm{GeV}$ range [32, 33, 45-48], but implies a lighter mostly singlet-like Higgs state $H_{1}$. 
Recent phenomenological constraints include, amongst others, upper bounds on the direct (spin independent) detection rate of dark matter by LUX [5]. In the NMSSM, the LSP (the dark matter candidate) is assumed to be the lightest neutralino, as in the MSSM. Its spin independent detection rate and relic density are computed with the help of micrOMEGAS_3 [43]. We apply the upper bounds of LUX and require a relic density inside a slightly enlarged WMAP/Planck window $[49,50] 0.107 \leq \Omega h^{2} \leq 0.131$ in order not to loose too many points in parameter space; the precise value of $\Omega h^{2}$ has little impact on the subsequent results.

In the Higgs sector we require a neutral CP-even state with a mass of $125.7 \pm 3 \mathrm{GeV}$ allowing for theoretical and parametric uncertainties of the mass calculation; we used $173.1 \mathrm{GeV}$ for the top quark mass. Its signal rates should comply with the essentially SMlike signal rates in the channels measured by ATLAS/CMS/Tevatron. These measurements can be combined leading to $95 \%$ confidence level (CL) contours in the planes of Higgs production via (gluon fusion and $\mathrm{ttH}$ ) - (vector boson fusion and associate production with $\mathrm{W} / \mathrm{Z}$ ), separately for Higgs decays into $\gamma \gamma$, ZZ or WW and $b \bar{b}$ or $\tau^{+} \tau^{-}$. We require that the signal rates for a Higgs boson in the above mass range are within all three $95 \%$ confidence level contours derived in [51].

The application of constraints from unsuccessful searches for sparticles at the first run of the LHC is more delicate: these bounds depend on all parameters of the model via the masses and couplings (and the resulting decay cascades) of all sparticles. However, it is possible to proceed as follows, using the most constraining searches for gluinos and squarks of the first generation in events with jets and missing $E_{T}$ : for heavy squarks and/or gluinos the production cross sections are so small that these points in parameter space are not excluded independently of the squark/gluino decay cascades. On the other hand, relatively light squarks and/or gluinos are excluded independently of their decay cascades. In between these regions defined in the planes of squark/gluino masses or $m_{0} / M_{1 / 2}$, exclusion does depend on their decays, in particular on the presence of a light singlino-like LSP at the end of the cascades $[52,53]$.

The boundaries between these three regions were obtained with the help of the analysis of some hundreds of points in parameter space: events were generated by MadGraph/MadEvent [54] which includes Pythia 6.4 [55] for showering and hadronisation. The sparticle branching ratios are obtained with the help of the code NMSDECAY [56] (based on SDECAY [57]), and are passed to Pythia. The output in StdHEP format is given to CheckMATE [58] which includes the detector simulation DELPHES [59] and compares the signal rates to constraints in various search channels of ATLAS and CMS. Corresponding results will be presented in section 3 .

Other constraints from $b$-physics, LEP (from Higgs searches and invisible Z decays) and the LHC (on heavy Higgs bosons decaying into $\tau^{+} \tau^{-}$) are applied as in NMSSMTools_4.2.1 [41, 42], leaving aside the muon anomalous magnetic moment.

Since the fundamental parameters of the model are the masses and couplings at the GUT scale, it makes sense to ask in how far these have to be tuned relative to each other in order to comply with the SM-like Higgs mass and the non-observation of sparticles at 
the LHC. To this end we consider the usual measure of fine-tuning [60]

$$
F T=\operatorname{Max}\left\{\left|\frac{\partial \ln \left(M_{Z}\right)}{\partial \ln \left(p_{i}^{G U T}\right)}\right|\right\}
$$

where $p_{i}^{G U T}$ denote all dimensionful and dimensionless parameters (Yukawa couplings, mass terms and trilinear couplings) at the GUT scale. FT is computed numerically in NMSSMTools_4.2.1 following the method described in [61] where details can be found.

We have scanned the parameter space of the NUH-NMSSM given in (2.9) using a Markov Chain Monte Carlo (MCMC) technique. In addition to the phenomenological constraints discussed above we require the absence of Landau singularities of the running Yukawa couplings below the GUT scale, and the absence of deeper unphysical minima of the Higgs potential with at least one vanishing vev $v_{u}, v_{d}$ or $s$. Bounds on the dimensionful parameters follow from the absence of too large fine-tuning; we imposed $F T<1000$. Finally we obtained $\sim 3.2 \times 10^{6}$ valid points in parameter space within the following ranges of the parameters $(2.9)$ :

$$
\begin{aligned}
& 1 \times 10^{-6} \leq \lambda \leq 0.722, \quad-0.08 \leq \kappa \leq 0.475, 1.42 \leq \tan \beta \leq 60.3, \\
&-537 \mathrm{GeV} \leq \mu_{\mathrm{eff}} \leq 753 \mathrm{GeV}, \quad-19 \mathrm{TeV} \leq A_{\lambda} \leq 8.5 \mathrm{TeV}, \quad-1.3 \mathrm{TeV} \leq A_{\kappa} \leq 5.3 \mathrm{TeV}, \\
& 0 \leq m_{0} \leq 4.4 \mathrm{TeV}, \quad 0.1 \mathrm{TeV} \leq M_{1 / 2} \leq 3.1 \mathrm{TeV}, \quad-6.6 \mathrm{TeV} \leq A_{0} \leq 8.1 \mathrm{TeV} .
\end{aligned}
$$

The fact that the upper bounds on the dimensionful parameters are distinct originates from the different impact of these parameters on the fine-tuning, which is often dominated by the universal gaugino mass parameter $M_{1 / 2}$.

\section{Impact of LHC constraints on squark/gluino masses and fine-tuning}

Strong constraints on parameter spaces of SUSY extensions of the SM come from searches for gluinos $\tilde{g}$ and squarks $\tilde{q}$ of the first generation in events with jets and missing $E_{T}[3,4]$. In [3] exclusion limits for MSUGRA/CMSSM models have been given in the $m_{0}-M_{1 / 2}$ and $M_{\tilde{g}}-m_{\tilde{q}}$ planes for $\tan \beta=30, A_{0}=-2 m_{0}$ and $\mu>0$.

As a result of the simulations described in the previous section we found that the 95\% CL upper limits on signal events in [3] lead to exclusion limits in the $m_{0}-M_{1 / 2}$ or $M_{\tilde{g}}-m_{\tilde{q}}$ planes in the NUH-NMSSM which are very similar to the CMSSM if the LSP is bino-like, but can be alleviated in the presence of a light singlino-like LSP at the end of the cascades $[52,53]$. Still, even with a singlino-like LSP, certain regions in these planes are always excluded.

In figure 1 we show the $m_{0}-M_{1 / 2}$ and $M_{\tilde{g}}-m_{\tilde{q}}$ planes in the NUH-NMSSM and indicate in green the regions allowed by the 95\% CL upper limits on signal events (practically identical to the ones given in [3]), in blue the regions possibly allowed in the presence of a singlino-like LSP, and in red the regions which are always excluded. Note that, in contrast to the MSSM, the limit $m_{0} \rightarrow 0$ is always possible for all $M_{1 / 2}$ : in the MSSM this region is limited by the appearance of a stau LSP. In the NMSSM a singlino-like LSP can always be lighter than the lightest stau, and its relic density can be reduced to the WMAP/Planck 

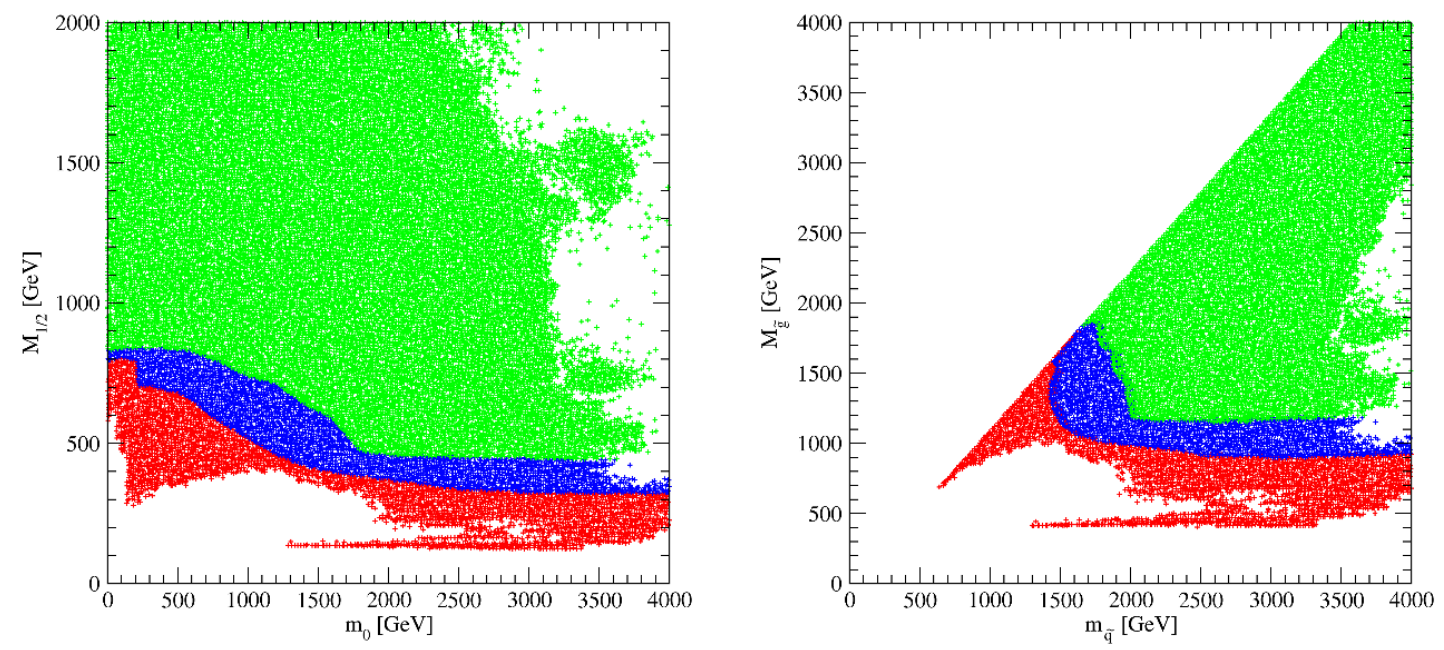

Figure 1. The $m_{0}-M_{1 / 2}$ and $M_{\tilde{g}}-m_{\tilde{q}}$ planes in the NUH-NMSSM. Green: regions allowed by the 95\% CL upper limits on signal events in [3], blue: regions allowed in the presence of a singlino-like LSP, red: regions which are always excluded.
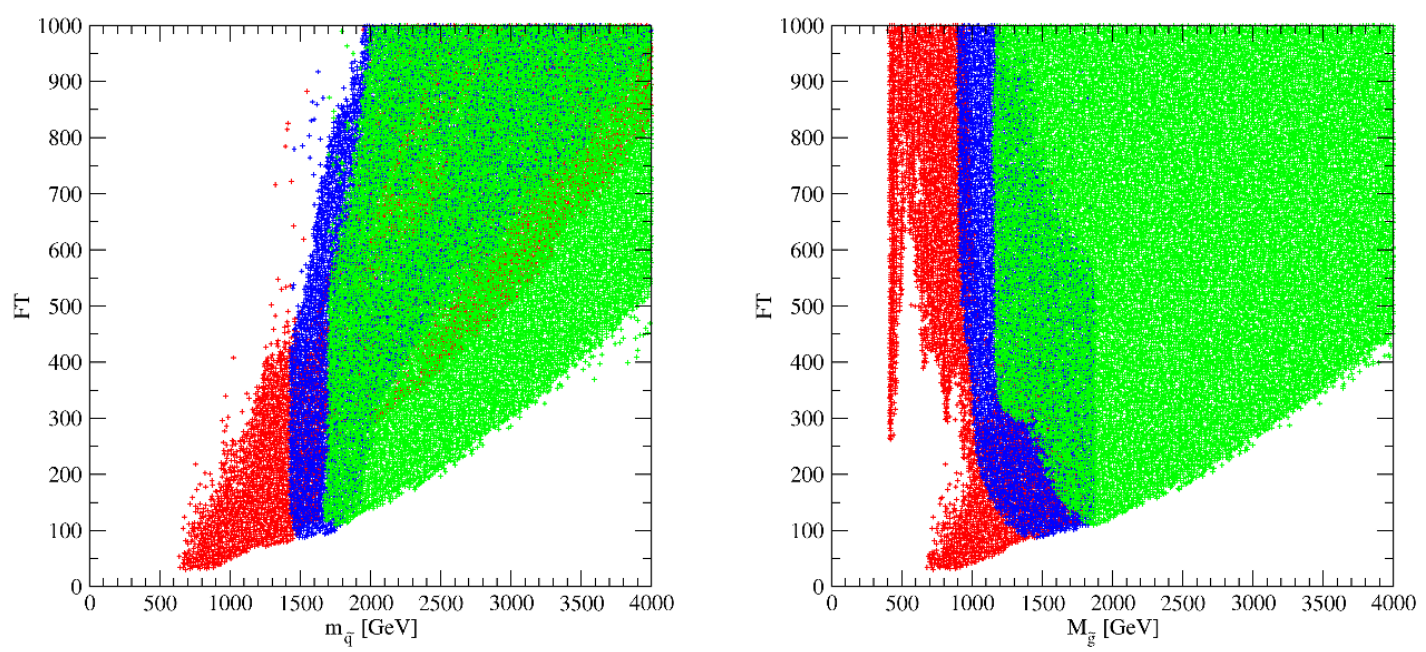

Figure 2. FT as defined in (2.10) as function of the squark and gluino masses, and the impact of the LHC constraints in the same color coding as in figure 1.

window through singlino-stau coannihilation as in the fully constrained NMSSM $[62,63]$ or through narrow resonances implying specific NMSSM light Higgs states [34, 67-69]. (The combined constraints from the Higgs sector and the nature of the LSP lead to discontinuities in the allowed parameter space for small $m_{0}$.)

These lower bounds on the squark and gluino masses dominate the lower bounds on the fine-tuning $F T$ defined in (2.10). In figure 2 we show $F T$ as function of the squark and gluino masses, and the impact of the LHC constraints in the same color coding as in figure 1. 

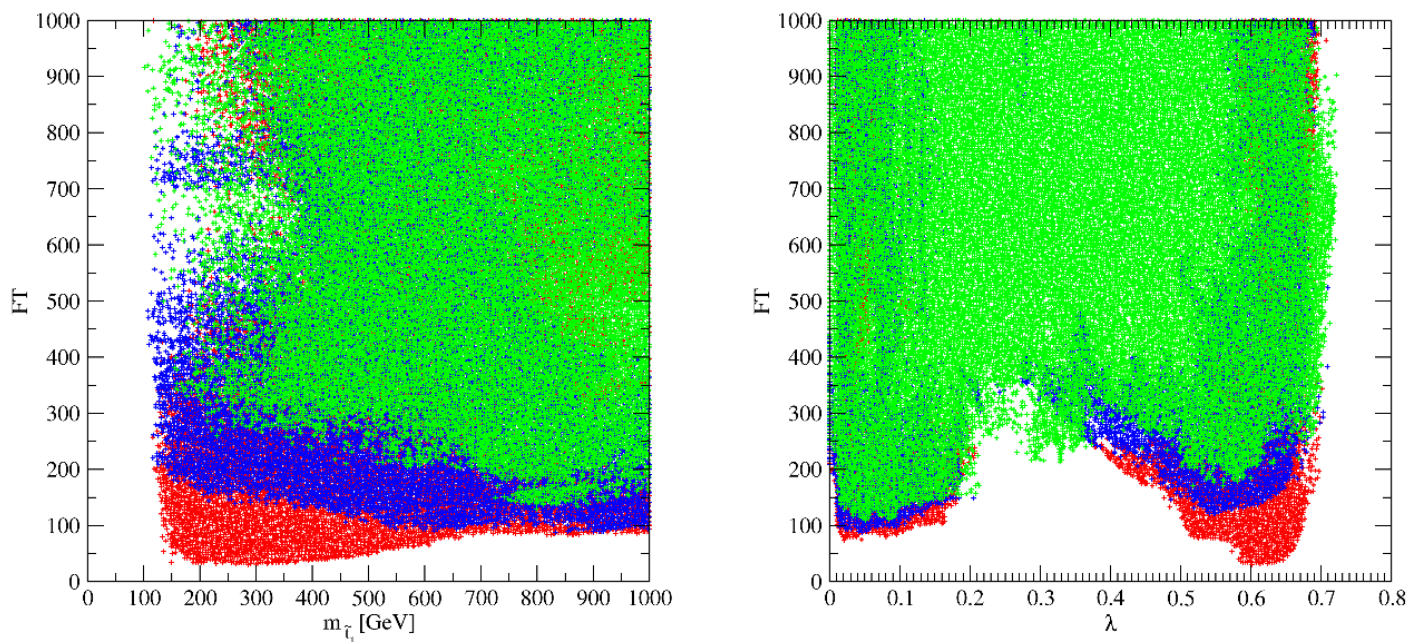

Figure 3. Left: $F T$ as function of the $m_{\tilde{t}_{1}}$. Right: $F T$ as function of $\lambda$ at the SUSY scale. The color coding is as in figure 1.

We see that the LHC forbidden red region increases the lower bound on FT from $\sim 20$ to $F T \gtrsim 80$; the NMSSM-specific alleviation (blue region) has a minor impact on $F T$. The dominant contribution to $F T$ in (2.10) originates typically from $M_{1 / 2}$ (i.e. the gluino mass at the GUT scale), or from the soft Higgs mass term $m_{H_{u}}^{2}$. If one requires unification of $m_{H_{u}}$ and $m_{H_{d}}$ with $m_{0}$ as in [30], FT is considerably larger ( $\left.\gtrsim 400\right)$. In the MSSM after imposing LHC constraints on squark and gluino masses, defining $F T$ with respect to parameters at the GUT scale and allowing for non-universal Higgs mass terms at the GUT scale as in [64] — one finds $F T \gtrsim 1000$. The much lower value of $F T$ in the NUH-NMSSM coincides with the result in [65].

The impact of $M_{1 / 2}$ on $F T$ is actually indirect: heavy gluinos lead to large radiative corrections to the stop masses which, in turn, lead to large radiative corrections to the soft Higgs mass terms. Therefore, if one defines $F T$ with respect to parameters at a lower scale, low $F T$ is typically related to light stops. On the left-hand side of figure 3 we show $F T$ as function of the mass $m_{\tilde{t}_{1}}$ of the lightest stop. We see that, without imposing LHC constraints on squark and gluino masses, the lower bound on FT (still with respect to parameters at the GUT scale) would increase slightly with $m_{\tilde{t}_{1}}$, but with LHC constraints the lower bound on FT depends weakly on (decreases only slightly with) $m_{\tilde{t}_{1}}$.

In the MSSM, the measured mass of the SM-like Higgs $H_{S M}$ requires relatively heavy stops and/or a Higgs-stop trilinear coupling $A_{t}$, which also contribute to FT. In the NMSSM (recall that, in the scenario considered here, $H_{S M}=H_{2}$ ) large radiative corrections to the SM-like Higgs mass $m_{H_{S M}}$ are not required, since the SM-like Higgs mass can be pushed upwards either through a positive tree level contribution $\sim \lambda^{2} \sin ^{2} 2 \beta$ [44], or through mixing with a lighter Higgs state $H_{1}[66]$ which does not require large values of $\lambda$ [71]. (In the latter scenario too large values of $\lambda$, i.e. a too large $H_{1}-H_{S M}$ mixing angle, can imply an inacceptable reduction of the signal rates of $H_{S M}$ at the LHC and/or lead to the violation of LEP constraints on $H_{1}$.) 

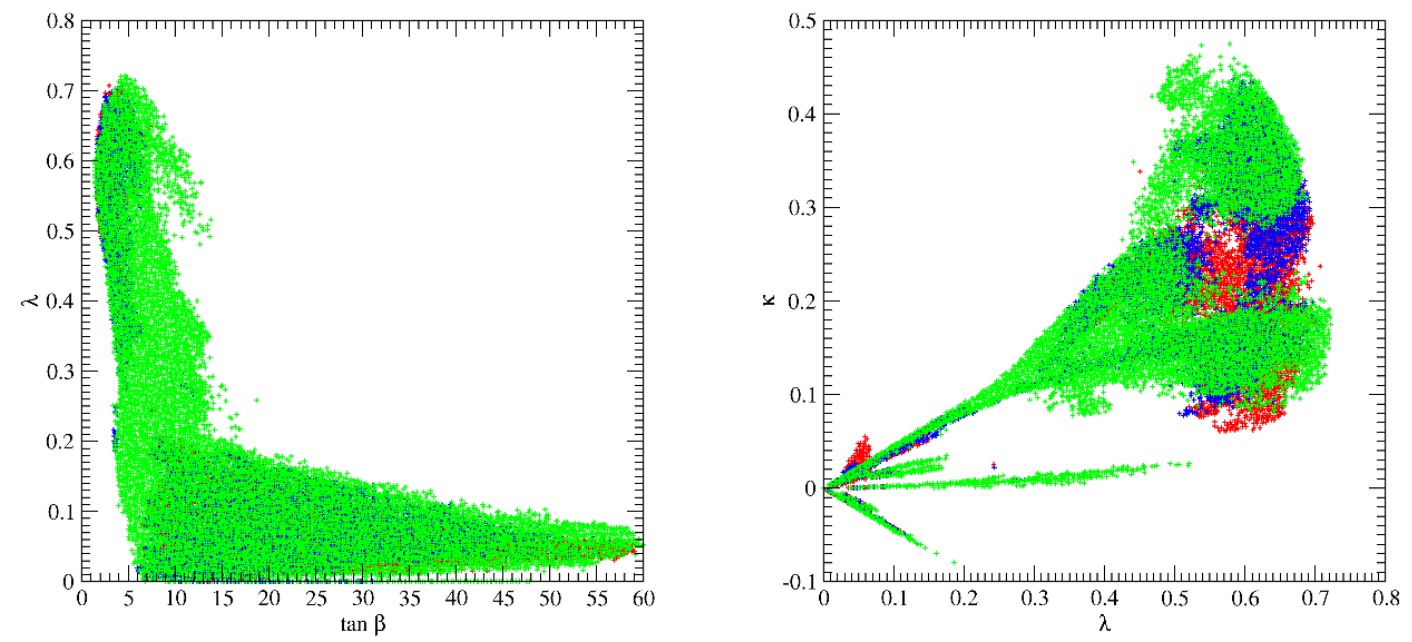

Figure 4. Left: $\lambda$ as function of $\tan \beta$. Right: $\kappa$ as function of $\lambda$. The colors are as in figure 1 .

On the right-hand side of figure 3 we show FT as function of $\lambda$. We see that without imposing LHC constraints on squark and gluino masses - the minimum of FT would indeed be assumed for $\lambda \sim 0.6$ related to the tree level contribution $\sim \lambda^{2} \sin ^{2} 2 \beta$ to $m_{H_{S M}}$. Including LHC constraints, local minima of $F T$ exist both for $\lambda \approx 0.6$ and $\lambda \approx 0.1$.

Since the increase of the SM-like Higgs mass with the help of the tree level contribution $\sim \lambda^{2} \sin ^{2} 2 \beta$ is effective only for large $\lambda$ but relatively low $\tan \beta$, these regions are typically correlated which is clarified on the left hand side of figure 4 . On the right hand side we show the correlations between $\lambda$ and $\kappa$ which shows that larger $\kappa$ are typically related to larger $\lambda$.

Herewith we conclude the discussion of the impact of LHC constraints on FT and the corresponding correlations with other parameters.

\section{Properties of dark matter}

Besides the enlarged Higgs sector, the enlarged neutralino sector of the NMSSM can have a significant phenomenological impact. The LSP (the lightest neutralino $\chi_{1}^{0}$ ) can have a dominant singlino component and still be an acceptable candidate for dark matter. Its relic density can be reduced to fit in the WMAP/Planck window, amongst others, via the exchange of NMSSM-specific CP-even or CP-odd Higgs scalars in the s-channel [34, 67-69], whereas its direct detection cross section can be very small.

The latter feature is clarified in figure 5 where we show the spin-independent $\chi_{1}^{0}$-nucleon cross section (after imposing constraints from the LUX experiment [5]) as function of $M_{\chi_{1}^{0}}$. We focus on $\chi_{1}^{0}$ masses below $100 \mathrm{GeV}$ since no additional interesting features appear for larger $M_{\chi_{1}^{0}}$, but the region of small $M_{\chi_{1}^{0}}$ exhibits structures which ask for explanations.

In figure 5 we have indicated the expected neutrino background to future direct dark matter detection experiments from [70] as a black line; it will be difficult to impossible to measure $\chi_{1}^{0}$-nucleon cross section smaller than this background. Unfortunately we see that 


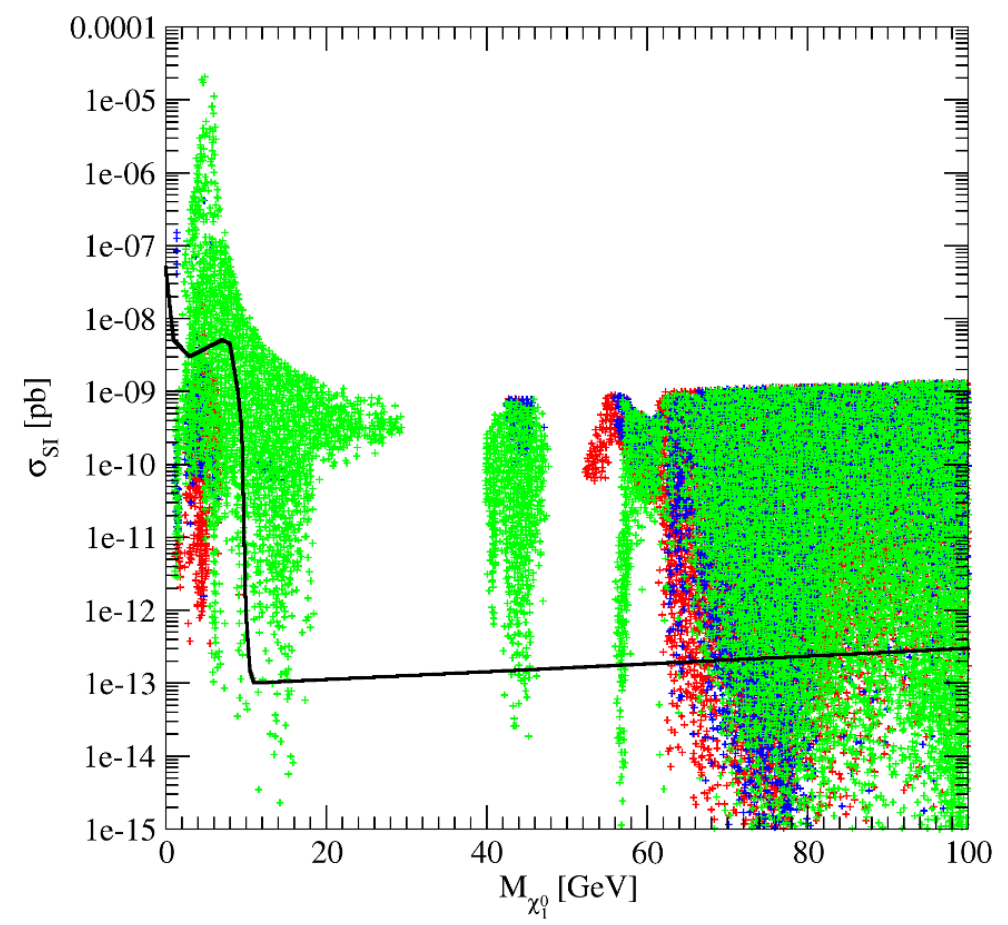

Figure 5. The spin-independent $\chi_{1}^{0}$-nucleon cross section $\sigma_{\mathrm{SI}}$ (after imposing constraints from the LUX experiment [5]) as function of $M_{\chi_{1}^{0}}$, focussing on $M_{\chi_{1}^{0}}<100 \mathrm{GeV}$. The black line indicates the expected neutrino background to future direct dark matter detection experiments (from [70]). The colors are as in figure 1.

significant regions in the NUH-NMSSM parameter space - notably for $M_{\chi_{1}^{0}} \lesssim 10 \mathrm{GeV}$ or $M_{\chi_{1}^{0}} \gtrsim 60 \mathrm{GeV}$ - may lead to such small cross sections.

Small $\chi_{1}^{0}$-nucleon cross sections originate from a large singlino component of $\chi_{1}^{0}$. Its singlino component $N_{15}^{2}$ is shown as function of $M_{\chi_{1}^{0}}$ in figure 6 .

Different regions of $M_{\chi_{1}^{0}}$ correspond to different dominant diagrams contributing to $\chi_{1}^{0}-\chi_{1}^{0}$ annihilation before its freeze-out. For small $M_{\chi_{1}^{0}} \lesssim 30 \mathrm{GeV}$ these are the exchange of NMSSM-specific CP-even or CP-odd Higgs scalars with masses $\approx 2 M_{\chi_{1}^{0}}$ in the s-channel, with couplings originating from the cubic $S^{3}$ term proportional to $\kappa$ in the superpotential. For $M_{\chi_{1}^{0}} \sim 40-48 \mathrm{GeV}, \chi_{1}^{0}-\chi_{1}^{0}$ annihilation is dominated by $Z$-exchange in the s-channel. The larger is the singlino component of $\chi_{1}^{0}$, the closer $M_{\chi_{1}^{0}}$ has to be to $M_{Z} / 2$ in order to compensate for the smaller coupling. For $M_{\chi_{1}^{0}} \sim 55-62 \mathrm{GeV}, \chi_{1}^{0}-\chi_{1}^{0}$ annihilation is

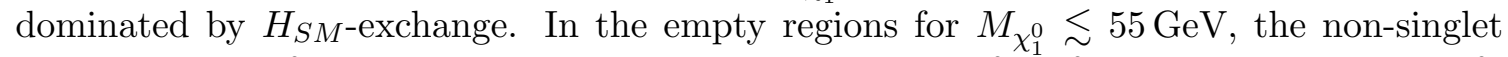
components of $\chi_{1}^{0}$ would have to be so large for successful $\chi_{1}^{0}-\chi_{1}^{0}$ annihilation that the $\chi_{1}^{0}$ nucleon cross section would violate constraints from LUX. For $M_{\chi_{1}^{0}} \gtrsim 62 \mathrm{GeV} \chi_{1}^{0}$ can have sizeable bino and/or higgsino components allowing for numerous additional (e.g. MSSMlike) $\chi_{1}^{0}-\chi_{1}^{0}$ annihilation channels. 


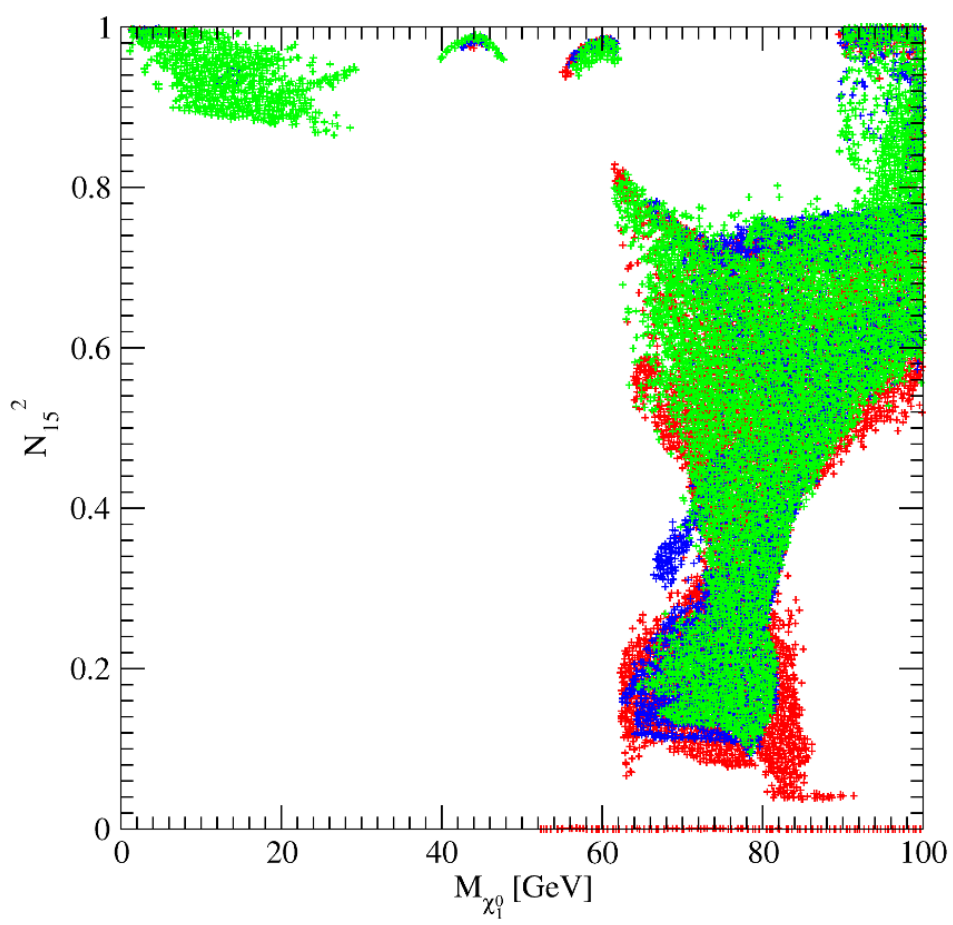

Figure 6. The $\chi_{1}^{0}$ singlino component (squared) as function of $M_{\chi_{1}^{0}}$. The colors are as in figure 1.

\section{Properties of the lighter Higgs boson $H_{1}$}

In this paper we focus on scenarios where mixing of the SM-like Higgs boson $H_{S M}$ with a lighter NMSSM-specific mostly singlet-like Higgs boson $H_{1}$ helps to increase the mass of $H_{S M}$. This is possible even for relatively small values of $\lambda \approx 0.1$ and moderate to large values of $\tan \beta[71]$.

However, the $H_{S M}-H_{1}$ mixing angle must not be too large: it leads to a reduction of the $H_{S M}$ couplings to electroweak gauge bosons and quarks, hence to a reduction of its production cross section at the LHC. These must comply with the measured signal rates, for which we require values inside the $95 \% \mathrm{CL}$ contours of [51]. Moreover, for $M_{H_{1}} \lesssim 114 \mathrm{GeV}$, $H_{1}$ must satisfy constraints from Higgs searches at LEP [72].

Hence the question is whether there are realistic prospects for the discovery of $H_{1}$ at the LHC [73]. First we consider the case where $H_{1}$ does not decay dominatly into pairs of lighter NMSSM-specific CP-odd Higgs bosons. The branching fractions of $H_{1}$ into $Z Z$ and $W^{+} W^{-}$ are small, both due to its smaller mass and its reduced couplings to $Z Z$ and $W^{+} W^{-}$.

The branching fraction of $H_{1}$ into $\gamma \gamma$ can be considerably larger than the one of a SMlike Higgs boson of the same mass [71, 74], both due to a possible reduction of its width into the dominant $b \bar{b}$ channel through mixing, and/or due to additional (higgsino-like) chargino loops contributing to the $H_{1}-\gamma \gamma$ coupling where the latter involve the NMSSM-specific coupling $\lambda[75,76]$. 


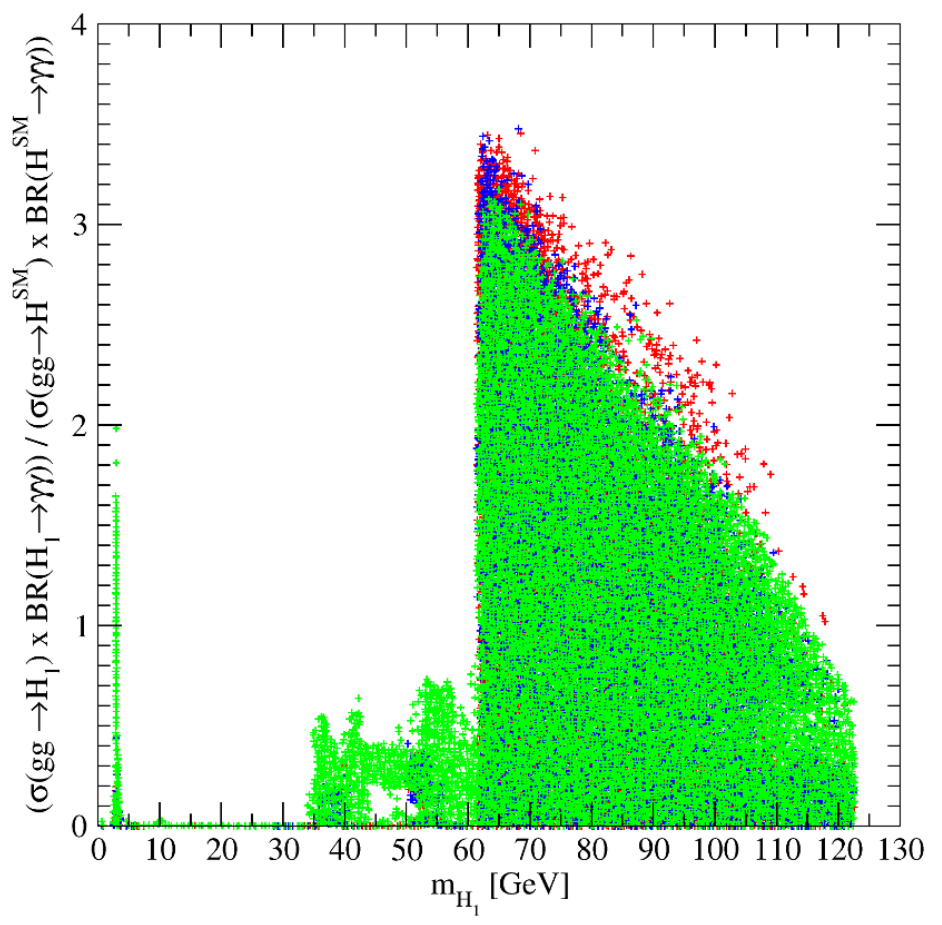

Figure 7. The $H_{1}$ signal rate in gluon fusion and the $\gamma \gamma$ channel relative to a SM-like Higgs boson $H^{S M}$ of the same mass. The color code is as in figure 1.

However, due to the reduced coupling of $H_{1}$ to SM particles, its production cross section $\sigma_{H_{1}}$ is smaller than the one of a SM Higgs boson $H^{S M}$ of the same mass. Hence one has to consider the reduced signal rate $\sigma_{H_{1}} \times B R\left(H_{1} \rightarrow \gamma \gamma\right) /\left(\sigma_{H^{S M}} \times B R\left(H^{S M} \rightarrow \gamma \gamma\right)\right)[71$, $74,77,78]$ which is shown for production via gluon fusion in figure 7 .

We see that the signal rate can be about 3.5 times larger than the one of a SMlike Higgs boson of a mass of $\sim 60 \mathrm{GeV}$. The absence of points with large signal rates for $M_{H_{1}} \lesssim 60 \mathrm{GeV}$ follows from the constraints on the signal rates of $H_{S M}$ : for $M_{H_{1}} \lesssim 60 \mathrm{GeV}$, $H_{S M}$ could decay into a pair of $H_{1}$ bosons, and this decay channel is easily dominant if kinematically allowed. The corresponding reductions of the other $H_{S M}$ branching fractions would be incompatible with its measured signal rates. (The possible enhancement of the signal rate for $M_{H_{1}} \lesssim 3.5 \mathrm{GeV}$ originates from the absence of decays into $b \bar{b}$ and $\tau^{+} \tau^{-}$, which makes it very sensitive to relative enhancements of the width into $\gamma \gamma$ via chargino loops.) For $M_{H_{1}} \gtrsim 110 \mathrm{GeV}$, some points with a reduced signal rate $\gtrsim 0.5$ could actually already be excluded by limits from CMS in [79] depending, however, on the relative contribution of gluon fusion to the expected signal rate in this mass range. On the other hand it is clear that, for $M_{H_{1}} \sim M_{Z}$, the $H_{1} \rightarrow \gamma \gamma$ channel faces potentially large backgrounds from fake photons from $Z \rightarrow e^{+} e^{-}$decays.

For the $b \bar{b}$ and $\tau^{+} \tau^{-}$final states we found that due to the reduction of the production cross section and the reduction of the couplings (i.e. branching fractions) of $H_{1}$ its reduced signal rates in gluon fusion, vector boson fusion and associate production with $Z / W$ are 

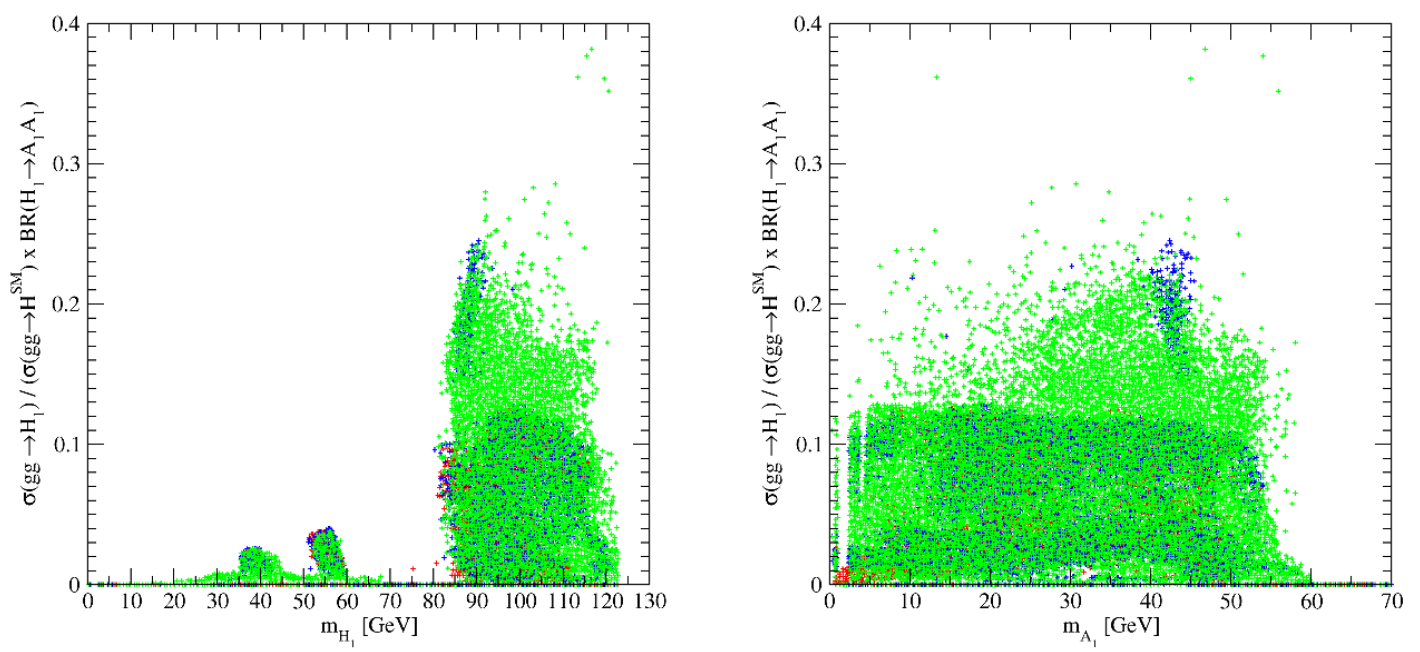

Figure 8. $\sigma_{H_{1}}(g g F) / \sigma_{H^{S M}}(g g F) \times B R\left(H_{1} \rightarrow A_{1} A_{1}\right)$ as function of $M_{H_{1}}$ (left) and $M_{A_{1}}$ (right). The color coding is as in figure 1 .

always below 0.3 for $M_{H_{1}} \lesssim 114 \mathrm{GeV}$, and still below 0.6 for $114 \mathrm{GeV} \lesssim M_{H_{1}} \lesssim 126 \mathrm{GeV}$; hence we will not further analyse these channels (also plagued by the absence of narrow peaks in the invariant mass of the final states).

Another possibility is that $H_{1}$ decays dominantly into pairs of light NMSSM-specific CP-odd Higgs bosons $A_{1}$ (see [80] and refs. therein). If this channel is open, the corresponding branching fraction $B R\left(H_{1} \rightarrow A_{1} A_{1}\right)$ can vary from 0 to 1 for all $M_{H_{1}}$ and $M_{A_{1}}$. However, the production cross section of $H_{1}$ is always reduced relative to the one of a SMlike Higgs boson $H^{S M}$ of the same mass. Focussing again on gluon fusion, we show in figures 8 the $B R\left(H_{1} \rightarrow A_{1} A_{1}\right)$ multiplied by the reduced $H_{1}$ production cross section (relative to the one of a SM-like Higgs boson $H^{S M}$ of the same mass) as function of $M_{H_{1}}$ and $M_{A_{1}}$.

The dominant decay branching fractions of $A_{1}$ are very similar to the ones of a SMlike Higgs boson of the same mass, i.e. dominantly into $b \bar{b}$ and $\tau^{+} \tau^{-}$if kinematically allowed. These unconventional channels $H \rightarrow A_{1} A_{1} \rightarrow \ldots$ have been searched for at LEP by OPAL [81-83], DELPHI [84] and ALEPH [85]. The corresponding constraints are taken into account in NMSSMTools, and explain the absence of sizeable signal rates for $M_{H_{1}} \lesssim 80 \mathrm{GeV}$. For $M_{H_{1}} \gtrsim 86 \mathrm{GeV}$ and, simultaneously, $0.25 \mathrm{GeV} \lesssim M_{A_{1}} \lesssim 3.55 \mathrm{GeV}$, first LHC analyses by CMS [86] have lead to upper limits on the signal cross section for $H \rightarrow A_{1} A_{1} \rightarrow 4 \mu$ which exclude some of the points in this range of $M_{A_{1}}$.

For heavier $A_{1}$ leading to dominant $b \bar{b}$ and/or $\tau^{+} \tau^{-}$decays, analyses of possible signals are certainly more difficult. At least we find that, for $M_{H_{1}} \gtrsim 80 \mathrm{GeV}$, production cross sections times branching fractions can be relatively large without violating present constraints, which should motivate future analyses of these channels.

Concerning the signal rates of the SM-like Higgs boson $\mathrm{H}_{2}$ we remark that all values allowed by the $95 \%$ confidence level contours in [51] in the planes of Higgs production via (gluon fusion and $\mathrm{ttH}$ ) - (vector boson fusion and associate production with $\mathrm{W} / \mathrm{Z}$ ) for Higgs decays into $\gamma \gamma$, ZZ+WW and $b \bar{b}+\tau^{+} \tau^{-}$have been found by our scan. 
Also possible are decays of $H_{2}$ into pairs of light CP-even or CP-odd states $H_{1}$ or $A_{1}$. They are limited by the SM-like signal rates of $H_{2}$, but branching fractions of up to $40 \%$ are still allowed.

\section{Conclusions and outlook}

In spite of the recent constraints on the mass and the signal rates at the LHC on a SM-like Higgs boson, upper bounds on signal rates generated by first generation squarks and gluinos and upper bounds on dark matter - nucleus cross sections we have seen that large ranges of the parameter space of the NUH-NMSSM remain viable. Here we confined ourselves to ranges with an additional lighter NMSSM-specific Higgs boson $H_{1}$ whose mixing with the SM-like Higgs boson can help to explain the mass of the latter of about $126 \mathrm{GeV}$ with relatively low finetuning (besides large values of $\lambda$ which, as can be seen from figure 3, are also part of this region). Within this scenario, bounds from squark/gluino searches dominate the lower bounds on fine-tuning which remain, on the other hand, considerably smaller than in the (NUHM-)MSSM and more constrained versions of the NMSSM.

The mass of the LSP is barely constrained, up to some "holes" around 30 and $50 \mathrm{GeV}$, and can possibly be below $1 \mathrm{GeV}$. Due to its possibly dominant singlino component, its direct detection cross section can be considerably smaller than the neutrino background, which makes it compatible with all future null-results in direct (and actually also indirect) dark matter searches.

We have not discussed all possible NUH-NMSSM-specific phenomena at colliders, which would be beyond the scope of the present paper. Here we focussed on the properties of the lighter Higgs boson $H_{1}$, in particular on its signal rates in channels which are accessible at the LHC. These include the potentially promising diphoton decay channel, but also $H_{1}$-decays into a pair of even lighter $\mathrm{CP}$-odd bosons $A_{1}$. Albeit taking into account all present constraints on additional lighter Higgs bosons, wide ranges of $H_{1}$ and $A_{1}$ masses remain to be explored.

Amongst additional NUH-NMSSM-specific phenomena at colliders - induced by a singlino-like LSP and/or additional Higgs states - are possibly unconventional cascade decays of charginos and top- and bottom-squarks, which require additional studies. Future work will also be dedicated to the possibilities for and signatures of Higgs-to-Higgs decay cascades induced by heavier Higgs states in the NUH-NMSSM.

\section{Acknowledgments}

UE acknowledges support from the ERC advanced grant Higgs@LHC and from the European Union Initial Training Networks INVISIBLES (PITN-GA-2011-289442) and HiggsTools (PITN-GA-2012-316704). The authors acknowledge the support of France Grilles for providing computing resources on the French National Grid Infrastructure.

Open Access. This article is distributed under the terms of the Creative Commons Attribution License (CC-BY 4.0), which permits any use, distribution and reproduction in any medium, provided the original author(s) and source are credited. 


\section{References}

[1] ATLAS collaboration, Observation of a new particle in the search for the Standard Model Higgs boson with the ATLAS detector at the LHC, Phys. Lett. B 716 (2012) 1 [arXiv: 1207.7214] [INSPIRE].

[2] CMS collaboration, Observation of a new boson at a mass of $125 \mathrm{GeV}$ with the CMS experiment at the LHC, Phys. Lett. B 716 (2012) 30 [arXiv:1207.7235] [InSPIRE].

[3] ATLAS collaboration, Search for squarks and gluinos with the ATLAS detector in final states with jets and missing transverse momentum and $20.3 \mathrm{fb}^{-1}$ of $\sqrt{s}=8 \mathrm{TeV}$ proton-proton collision data, ATLAS-CONF-2013-047, CERN, Geneva Switzerland (2013).

[4] CMS collaboration, Search for new physics in the multijet and missing transverse momentum final state in proton-proton collisions at $\sqrt{s}=8 \mathrm{TeV}$, JHEP 06 (2014) 055 [arXiv: 1402.4770] [INSPIRE].

[5] LUX collaboration, D.S. Akerib et al., First results from the LUX dark matter experiment at the Sanford Underground Research Facility, Phys. Rev. Lett. 112 (2014) 091303 [arXiv: 1310.8214] [INSPIRE].

[6] H. Baer, V. Barger and A. Mustafayev, Implications of a $125 \mathrm{GeV}$ Higgs scalar for LHC SUSY and neutralino dark matter searches, Phys. Rev. D 85 (2012) 075010 [arXiv:1112.3017] [INSPIRE].

[7] A. Arbey, M. Battaglia, A. Djouadi, F. Mahmoudi and J. Quevillon, Implications of a 125 GeV Higgs for supersymmetric models, Phys. Lett. B 708 (2012) 162 [arXiv:1112.3028] [INSPIRE].

[8] O. Buchmueller et al., Higgs and supersymmetry, Eur. Phys. J. C 72 (2012) 2020 [arXiv:1112.3564] [INSPIRE].

[9] S. Akula, B. Altunkaynak, D. Feldman, P. Nath and G. Peim, Higgs boson mass predictions in SUGRA unification, recent LHC-7 results and dark matter, Phys. Rev. D 85 (2012) 075001 [arXiv: 1112.3645] [INSPIRE].

[10] M. Kadastik, K. Kannike, A. Racioppi and M. Raidal, Implications of the 125 GeV Higgs boson for scalar dark matter and for the CMSSM phenomenology, JHEP 05 (2012) 061 [arXiv: 1112.3647] [INSPIRE].

[11] J. Cao, Z. Heng, D. Li and J.M. Yang, Current experimental constraints on the lightest Higgs boson mass in the constrained MSSM, Phys. Lett. B 710 (2012) 665 [arXiv:1112.4391] [INSPIRE].

[12] J. Ellis and K.A. Olive, Revisiting the Higgs mass and dark matter in the CMSSM, Eur. Phys. J. C 72 (2012) 2005 [arXiv:1202.3262] [INSPIRE].

[13] A. Fowlie et al., The CMSSM favoring new territories: the impact of new LHC limits and a 125 GeV Higgs, Phys. Rev. D 86 (2012) 075010 [arXiv: 1206.0264] [INSPIRE].

[14] C. Beskidt, W. de Boer, D.I. Kazakov and F. Ratnikov, Constraints on supersymmetry from LHC data on SUSY searches and Higgs bosons combined with cosmology and direct dark matter searches, Eur. Phys. J. C 72 (2012) 2166 [arXiv:1207.3185] [INSPIRE].

[15] O. Buchmueller et al., The CMSSM and NUHM1 in light of 7 TeV LHC, Bs $\rightarrow \mu^{+} \mu^{-}$and XENON100 data, Eur. Phys. J. C 72 (2012) 2243 [arXiv:1207.7315] [InSPIRE]. 
[16] C. Strege et al., Global fits of the CMSSM and NUHM including the LHC Higgs discovery and new XENON100 constraints, JCAP 04 (2013) 013 [arXiv:1212.2636] [INSPIRE].

[17] J. Ellis, F. Luo, K.A. Olive and P. Sandick, The Higgs mass beyond the CMSSM, Eur. Phys. J. C 73 (2013) 2403 [arXiv:1212.4476] [INSPIRE].

[18] M.E. Cabrera, J.A. Casas and R.R. de Austri, The health of SUSY after the Higgs discovery and the XENON100 data, JHEP 07 (2013) 182 [arXiv:1212.4821] [INSPIRE].

[19] K. Kowalska, L. Roszkowski and E.M. Sessolo, Two ultimate tests of constrained supersymmetry, JHEP 06 (2013) 078 [arXiv: 1302.5956] [INSPIRE].

[20] T. Cohen and J.G. Wacker, Here be dragons: the unexplored continents of the CMSSM, JHEP 09 (2013) 061 [arXiv: 1305.2914] [INSPIRE].

[21] C. Beskidt, W. de Boer and D.I. Kazakov, A comparison of the Higgs sectors of the CMSSM and NMSSM for a 126 GeV Higgs boson, Phys. Lett. B 726 (2013) 758 [arXiv:1308.1333] [INSPIRE].

[22] S. Henrot-Versillé et al., Constraining supersymmetry using the relic density and the Higgs boson, Phys. Rev. D 89 (2014) 055017 [arXiv: 1309.6958] [INSPIRE].

[23] P. Bechtle et al., Constrained supersymmetry after the Higgs boson discovery: a global analysis with Fittino, PoS(EPS-HEP 2013) 313 [arXiv:1310.3045] [INSPIRE].

[24] D. Kim, P. Athron, C. Balázs, B. Farmer and E. Hutchison, Bayesian naturalness of the $C(N) M S S M$, arXiv:1312.4150 [INSPIRE].

[25] O. Buchmueller et al., The CMSSM and NUHM1 after LHC run 1, arXiv:1312.5250 [INSPIRE].

[26] J. Ellis, Supersymmetric fits after the Higgs discovery and implications for model building, Eur. Phys. J. C 74 (2014) 2732 [arXiv:1312.5426] [InSPIRE].

[27] J.F. Gunion, Y. Jiang and S. Kraml, The constrained NMSSM and Higgs near $125 \mathrm{GeV}$, Phys. Lett. B 710 (2012) 454 [arXiv:1201.0982] [INSPIRE].

[28] U. Ellwanger and C. Hugonie, Higgs bosons near $125 \mathrm{GeV}$ in the NMSSM with constraints at the GUT scale, Adv. High Energy Phys. 2012 (2012) 625389 [arXiv:1203.5048] [InSPIRE].

[29] G. Bélanger et al., Higgs bosons at 98 and $125 \mathrm{GeV}$ at LEP and the LHC, JHEP 01 (2013) 069 [arXiv: 1210.1976] [INSPIRE].

[30] BayesFitS Group collaboration, K. Kowalska et al., Constrained next-to-minimal supersymmetric Standard Model with a 126 GeV Higgs boson: a global analysis, Phys. Rev. D 87 (2013) 115010 [arXiv:1211.1693] [INSPIRE].

[31] C. Beskidt, W. de Boer and D.I. Kazakov, The impact of a 126 GeV Higgs on the neutralino mass, arXiv:1402.4650 [INSPIRE].

[32] Z. Kang, J. Li and T. Li, On naturalness of the MSSM and NMSSM, JHEP 11 (2012) 024 [arXiv: 1201.5305] [INSPIRE].

[33] J.-J. Cao, Z.-X. Heng, J.M. Yang, Y.-M. Zhang and J.-Y. Zhu, A SM-like Higgs near $125 \mathrm{GeV}$ in low energy SUSY: a comparative study for MSSM and NMSSM, JHEP 03 (2012) 086 [arXiv: 1202.5821] [INSPIRE].

[34] D.A. Vasquez et al., The $125 \mathrm{GeV}$ Higgs in the NMSSM in light of LHC results and astrophysics constraints, Phys. Rev. D 86 (2012) 035023 [arXiv:1203.3446] [INSPIRE]. 
[35] M. Perelstein and B. Shakya, XENON100 implications for naturalness in the MSSM, NMSSM and $\lambda$-supersymmetry model, Phys. Rev. D 88 (2013) 075003 [arXiv:1208.0833] [INSPIRE].

[36] K. Agashe, Y. Cui and R. Franceschini, Natural islands for a $125 \mathrm{GeV}$ Higgs in the scale-invariant NMSSM, JHEP 02 (2013) 031 [arXiv:1209.2115] [INSPIRE].

[37] T. Gherghetta, B. von Harling, A.D. Medina and M.A. Schmidt, The scale-invariant NMSSM and the $126 \mathrm{GeV}$ Higgs boson, JHEP 02 (2013) 032 [arXiv:1212.5243] [INSPIRE].

[38] T. Cheng, J. Li, T. Li and Q.-S. Yan, Natural NMSSM confronting with the LHC7-8, Phys. Rev. D 89 (2014) 015015 [arXiv: 1304.3182] [INSPIRE].

[39] J. Cao, F. Ding, C. Han, J.M. Yang and J. Zhu, A light Higgs scalar in the NMSSM confronted with the latest LHC Higgs data, JHEP 11 (2013) 018 [arXiv:1309.4939] [INSPIRE].

[40] U. Ellwanger and C. Hugonie, NMSPEC: a fortran code for the sparticle and Higgs masses in the NMSSM with GUT scale boundary conditions, Comput. Phys. Commun. 177 (2007) 399 [hep-ph/0612134] [INSPIRE].

[41] U. Ellwanger, J.F. Gunion and C. Hugonie, NMHDECAY: a fortran code for the Higgs masses, couplings and decay widths in the NMSSM, JHEP 02 (2005) 066 [hep-ph/0406215] [INSPIRE].

[42] U. Ellwanger and C. Hugonie, NMHDECAY 2.0: an updated program for sparticle masses, Higgs masses, couplings and decay widths in the NMSSM, Comput. Phys. Commun. 175 (2006) 290 [hep-ph/0508022] [INSPIRE].

[43] G. Bélanger, F. Boudjema, A. Pukhov and A. Semenov, MicrOMEGAs3: a program for calculating dark matter observables, Comput. Phys. Commun. 185 (2014) 960 [arXiv: 1305.0237] [INSPIRE].

[44] U. Ellwanger, C. Hugonie and A.M. Teixeira, The next-to-minimal supersymmetric Standard Model, Phys. Rept. 496 (2010) 1 [arXiv:0910.1785] [InSPIRE].

[45] L.J. Hall, D. Pinner and J.T. Ruderman, A natural SUSY Higgs near 126 GeV, JHEP 04 (2012) 131 [arXiv:1112.2703] [INSPIRE].

[46] U. Ellwanger, A Higgs boson near $125 \mathrm{GeV}$ with enhanced di-photon signal in the NMSSM, JHEP 03 (2012) 044 [arXiv: 1112.3548] [INSPIRE].

[47] A. Arvanitaki and G. Villadoro, A non Standard Model Higgs at the LHC as a sign of naturalness, JHEP 02 (2012) 144 [arXiv:1112.4835] [INSPIRE].

[48] S.F. King, M. Muhlleitner and R. Nevzorov, NMSSM Higgs benchmarks near 125 GeV, Nucl. Phys. B 860 (2012) 207 [arXiv:1201.2671] [InSPIRE].

[49] WMAP collaboration, G. Hinshaw et al., Nine-year Wilkinson Microwave Anisotropy Probe (WMAP) observations: cosmological parameter results, Astrophys. J. Suppl. 208 (2013) 19 [arXiv: 1212.5226] [INSPIRE].

[50] Planck collaboration, P.A.R. Ade et al., Planck 2013 results. XVI. Cosmological parameters, arXiv: 1303.5076 [INSPIRE].

[51] G. Bélanger, B. Dumont, U. Ellwanger, J.F. Gunion and S. Kraml, Global fit to Higgs signal strengths and couplings and implications for extended Higgs sectors, Phys. Rev. D 88 (2013) 075008 [arXiv: 1306 .2941] [INSPIRE]. 
[52] D. Das, U. Ellwanger and A.M. Teixeira, Modified signals for supersymmetry in the NMSSM with a singlino-like LSP, JHEP 04 (2012) 067 [arXiv:1202.5244] [INSPIRE].

[53] D. Das, U. Ellwanger and A.M. Teixeira, LHC constraints on $M_{1 / 2}$ and $m_{0}$ in the semi-constrained NMSSM, JHEP 04 (2013) 117 [arXiv:1301.7584] [INSPIRE].

[54] J. Alwall, M. Herquet, F. Maltoni, O. Mattelaer and T. Stelzer, MadGraph 5: going beyond, JHEP 06 (2011) 128 [arXiv: 1106.0522] [INSPIRE].

[55] T. Sjöstrand, S. Mrenna and P.Z. Skands, PYTHIA 6.4 physics and manual, JHEP 05 (2006) 026 [hep-ph/0603175] [INSPIRE].

[56] D. Das, U. Ellwanger and A.M. Teixeira, NMSDECAY: a fortran code for supersymmetric particle decays in the next-to-minimal supersymmetric Standard Model, Comput. Phys. Commun. 183 (2012) 774 [arXiv:1106.5633] [INSPIRE].

[57] M. Muhlleitner, A. Djouadi and Y. Mambrini, SDECAY: a fortran code for the decays of the supersymmetric particles in the MSSM, Comput. Phys. Commun. 168 (2005) 46 [hep-ph/0311167] [INSPIRE].

[58] M. Drees, H. Dreiner, D. Schmeier, J. Tattersall and J.S. Kim, CheckMATE: confronting your favourite new physics model with LHC data, arXiv:1312.2591 [INSPIRE].

[59] DELPHES 3 collaboration, J. de Favereau et al., DELPHES 3, a modular framework for fast simulation of a generic collider experiment, JHEP 02 (2014) 057 [arXiv:1307.6346] [INSPIRE].

[60] R. Barbieri and G.F. Giudice, Upper bounds on supersymmetric particle masses, Nucl. Phys. B 306 (1988) 63 [INSPIRE].

[61] U. Ellwanger, G. Espitalier-Noel and C. Hugonie, Naturalness and fine tuning in the NMSSM: implications of early LHC results, JHEP 09 (2011) 105 [arXiv:1107.2472] [INSPIRE].

[62] A. Djouadi, U. Ellwanger and A.M. Teixeira, The constrained next-to-minimal supersymmetric Standard Model, Phys. Rev. Lett. 101 (2008) 101802 [arXiv:0803.0253] [INSPIRE].

[63] A. Djouadi, U. Ellwanger and A.M. Teixeira, Phenomenology of the constrained NMSSM, JHEP 04 (2009) 031 [arXiv: 0811.2699] [INSPIRE].

[64] H. Baer et al., Radiative natural supersymmetry: reconciling electroweak fine-tuning and the Higgs boson mass, Phys. Rev. D 87 (2013) 115028 [arXiv:1212.2655] [INSPIRE].

[65] M.Y. Binjonaid and S.F. King, Naturalness of scale-invariant NMSSMs with and without extra matter, arXiv:1403.2088 [INSPIRE].

[66] U. Ellwanger and C. Hugonie, Masses and couplings of the lightest Higgs bosons in the $(M+1) S S M$, Eur. Phys. J. C 25 (2002) 297 [hep-ph/9909260] [INSPIRE].

[67] G. Bélanger, F. Boudjema, C. Hugonie, A. Pukhov and A. Semenov, Relic density of dark matter in the NMSSM, JCAP 09 (2005) 001 [hep-ph/0505142] [INSPIRE].

[68] C. Hugonie, G. Bélanger and A. Pukhov, Dark matter in the constrained NMSSM, JCAP 11 (2007) 009 [arXiv:0707.0628] [inSPIRE].

[69] G. Bélanger, C. Hugonie and A. Pukhov, Precision measurements, dark matter direct detection and LHC Higgs searches in a constrained NMSSM, JCAP 01 (2009) 023 [arXiv: 0811.3224] [INSPIRE]. 
[70] J. Billard, L. Strigari and E. Figueroa-Feliciano, Implication of neutrino backgrounds on the reach of next generation dark matter direct detection experiments, Phys. Rev. D 89 (2014) 023524 [arXiv: 1307.5458] [INSPIRE].

[71] M. Badziak, M. Olechowski and S. Pokorski, New regions in the NMSSM with a $125 \mathrm{GeV}$ Higgs, JHEP 06 (2013) 043 [arXiv: 1304.5437] [INSPIRE].

[72] ALEPH, DELPHI, L3, OPAL and LEP Working Group For HigGs Boson SEARCHES collaborations, S. Schael et al., Search for neutral MSSM Higgs bosons at LEP, Eur. Phys. J. C 47 (2006) 547 [hep-ex/0602042] [INSPIRE].

[73] G. Cacciapaglia, A. Deandrea, G.D. La Rochelle and J.-B. Flament, Searching for a lighter Higgs: parametrisation and sample tests, arXiv:1311.5132 [INSPIRE].

[74] U. Ellwanger, Enhanced di-photon Higgs signal in the next-to-minimal supersymmetric Standard Model, Phys. Lett. B 698 (2011) 293 [arXiv:1012.1201] [INSPIRE].

[75] K. Schmidt-Hoberg and F. Staub, Enhanced $h \rightarrow \gamma \gamma$ rate in MSSM singlet extensions, JHEP 10 (2012) 195 [arXiv: 1208.1683] [INSPIRE].

[76] K. Choi, S.H. Im, K.S. Jeong and M. Yamaguchi, Higgs mixing and diphoton rate enhancement in NMSSM models, JHEP 02 (2013) 090 [arXiv:1211.0875] [INSPIRE].

[77] J. Cao, Z. Heng, T. Liu and J.M. Yang, Di-photon Higgs signal at the LHC: a comparative study for different supersymmetric models, Phys. Lett. B 703 (2011) 462 [arXiv:1103.0631] [INSPIRE].

[78] S.F. King, M. Mühlleitner, R. Nevzorov and K. Walz, Natural NMSSM Higgs bosons, Nucl. Phys. B 870 (2013) 323 [arXiv: 1211.5074] [INSPIRE].

[79] CMS collaboration, Updated measurements of the Higgs boson at $125 \mathrm{GeV}$ in the two photon decay channel, CMS-PAS-HIG-13-001, CERN, Geneva Switzerland (2013).

[80] D.G. Cerdeno, P. Ghosh and C.B. Park, Probing the two light Higgs scenario in the NMSSM with a low-mass pseudoscalar, JHEP 06 (2013) 031 [arXiv:1301.1325] [INSPIRE].

[81] OPAL collaboration, G. Abbiendi et al., Decay mode independent searches for new scalar bosons with the OPAL detector at LEP, Eur. Phys. J. C 27 (2003) 311 [hep-ex/0206022] [INSPIRE].

[82] OPAL collaboration, G. Abbiendi et al., Search for neutral Higgs boson in CP-conserving and CP-violating MSSM scenarios, Eur. Phys. J. C 37 (2004) 49 [hep-ex/0406057] [INSPIRE].

[83] OPAL collaboration, G. Abbiendi et al., Search for a low mass CP odd Higgs boson in $e^{+} e^{-}$ collisions with the OPAL detector at LEP-2, Eur. Phys. J. C 27 (2003) 483 [hep-ex/0209068] [INSPIRE].

[84] DELPHI collaboration, J. Abdallah et al., Searches for neutral Higgs bosons in extended models, Eur. Phys. J. C 38 (2004) 1 [hep-ex/0410017] [INSPIRE].

[85] ALEPH collaboration, S. Schael et al., Search for neutral Higgs bosons decaying into four taus at LEP2, JHEP 05 (2010) 049 [arXiv: 1003.0705] [INSPIRE].

[86] CMS collaboration, Search for a non-Standard Model Higgs boson decaying to a pair of new light bosons in four-muon final states, Phys. Lett. B 726 (2013) 564 [arXiv:1210.7619] [INSPIRE]. 